\title{
Ebola Virus Entry into Host Cells: Identifying Therapeutic Strategies
}

\author{
Bethany A. Rhein ${ }^{1} \cdot$ Wendy J. Maury ${ }^{1}$
}

Published online: 1 July 2015

(C) Springer International Publishing AG 2015

\begin{abstract}
Filoviruses cause severe hemorrhagic fever in humans. The archetypal virus of this group, Ebola virus, is responsible for the current filovirus epidemic in West Africa. Filoviruses infect most mammalian cells, resulting in broad species tropism and likely contributing to rapid spread of virus throughout the body. A thorough understanding of filovirus entry events will facilitate the development of therapeutics against these critical steps in the viral life cycle. This review summarizes the current understanding of filovirus entry and discusses some of the recent advancements in therapeutic strategies that target entry.
\end{abstract}

Keywords Filoviruses · Ebola virus · Virus entry · Phosphatidylserine receptors $\cdot$ C-type lectins $\cdot$ NPC1 . Therapeutics $\cdot$ Proteolytic processing $\cdot$ Virus receptors

\section{Introduction}

Ebolavirus and Marburgvirus are members of the Filoviridae family of enveloped, negative-sense RNA viruses that cause severe hemorrhagic fever in humans and non-human primates (NHPs). There are four identified Ebolavirus species (Ebola

This article is part of the Topical Collection on Virology

Wendy J. Maury

wendy-maury@uiowa.edu

Bethany A. Rhein

bethany-rhein@uiowa.edu

1 Department of Microbiology, University of Iowa, 3-701 Bowen Science Building, 51 Newton Rd, Iowa City, IA 52242, USA virus [formerly Zaire Ebola virus; $\mathrm{EBOV}^{1}$ ], Bundibugyo virus, Sudan virus, and Taï Forest virus) that are associated with episodic hemorrhagic fever outbreaks in sub-Saharan Africa [1-3]. EBOV is the cause of the current West Africa epidemic, and over 26,000 individuals are thought to have been infected during this 18-month epidemic [3]. A fifth Ebolavirus member, found in the Philippines, is Reston virus that is pathogenic in nonhuman primates. No approved vaccines or antivirals are currently available against these viruses, and the current outbreak emphasizes the critical need for development of treatments.

Filoviruses infect a wide array of human primary cells and cell lines. Dendritic cells (DCs) and macrophages are major early and sustained targets of infection $[4,5]$. Viral replication in these cells is thought to amplify virus within the infected host, leading to systemic spread of a broad array of cell populations ranging from hepatocytes to endothelial cells to fibroblasts. Lymphocytes are one of the few cell types that are not productively infected by filoviruses in the body [6].

Recent findings identifying events associated with filovirus entry have provided insights into the uniquely complex entry mechanisms that this family of enveloped viruses uses. Entry is initiated by virion attachment at the plasma membrane, leading to a macropinocytosis-like internalization into endosomes. Once in the endosomal/lysosomal pathway, the viral glycoprotein (GP) undergoes proteolytic cleavage and structural rearrangements, facilitating interactions with host factors, including an essential intracellular receptor. Following this interaction, fusion of the viral and host membranes allows the release of the nucleocapsid core into the cytoplasm for viral replication. Elucidation of this series of events has revealed novel host-virus interactions and led to the identification of novel therapeutic targets against filoviruses. Here, we discuss the current understanding of

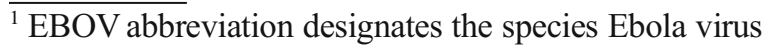


filovirus entry and recent developments in therapeutics that target the viral entry process.

\section{Filovirus Structure and Glycoprotein Expression}

Filoviruses form unique filamentous virus particles that are surrounded by a membrane acquired during viral budding from the host cell plasma membrane. Studies indicate that phosphatidylserine (PtdSer) is exposed on the outer leaflet of virion membranes, and the presence of PtdSer is important for virus attachment to cell surfaces $[7 \cdot, 8 \cdot]$. Within the virion, the RNA genome is surrounded and protected by the nucleocapsid complex composed of the nucleoprotein (NP), VP30, and VP35 [9]. The viral polymerase is thought to be recruited to this complex by interactions with VP35 and VP30 [10, 11]. VP40 and VP24, the major and minor matrix proteins, respectively, control virus morphogenesis, studding the inner leaflet of the viral membrane. More recently, these structural proteins have also been implicated in the regulation of filovirus RNA synthesis [12]. The viral GP is the only virally encoded protein present on the outer surface of virions and mediates virus-host membrane fusion.

Filovirus GP is a class I viral membrane fusion GP that is similar to HIV-1 Env and influenza virus hemagglutinin (HA) (recently reviewed [13]). The major product expressed by the EBOV GP gene is a secreted, soluble GP (sGP) [14]. The function of sGP is still unclear, but it is thought to be important in viral immune evasion (recently reviewed [15]). Full length, membrane-associated EBOV GP is produced by an RNA frame shift that results in the insertion of a nontemplated adenosine residue during transcriptional RNA editing [16]. In contrast, the Marburgvirus GP gene directly encodes membrane-associated GP, and an equivalent soluble form of Marburgvirus GP is not thought to not be expressed [17]. Mature filoviral GPs are formed by post-translational furin cleavage of the proprotein, producing the disulfidelinked heterodimer composed of GP1 and GP2 [18]. The GP1 subunit is required for receptor interactions and transmembrane-associated GP2 is required for membrane fusion. Like other class I viral membrane fusion GPs, filovirus GPs are found on virions as trimers. Crystal structures of both Ebolavirus species and Marburgvirus GP ectodomains have been solved [19, 20, 21••]. GP forms a chalice-like shape with a trimer of heterodimers of GP1/ GP2, where GP2 is the base and GP1 is the cup.

Filovirus GP1 has four distinct domains: base, receptorbinding domain (RBD), glycan cap, and mucin-like domain (MLD). The base interacts with GP2, providing structural support for the other domains. Residues within the RBD interact with an intracellular cellular receptor, Niemann-Pick C1 (NPC1) within the late endosomal/lysosomal compartments. The MLD and glycan cap are heavily glycosylated with $N$ - linked glycans and the MLD also contains as many as $80 \mathrm{O}$ linked glycans [19, 22, 23•]. Glycans of GP1 are important for shielding the GP from neutralizing antibodies [23•, 24-26].

Unlike many class I viral GPs, the filovirus GP2 fusion peptide resides in a loop near the $\mathrm{N}$-terminal sequence of GP2 rather than directly at the N-terminus. The 45 amino acid EBOV fusion loop is clamped by a disulfide bond and has a membrane-seeking hydrophobic sequence at the tip of the loop [27-30]. Within the pre-fusion form of GP, the hydrophobic loop sequences are protected by residues on an adjacent GP1 subunit until fusion events unfold. In addition to the fusion loop, GP2 contains two canonical helical repeats in its ectodomain as well as a transmembrane domain and a short cytoplasmic tail.

\section{Cell Surface Proteins Involved in Viral Attachment}

Several cell surface proteins have been identified to mediate attachment of filoviruses, and evidence suggests that these same molecules are responsible for virus entry into the endosome. C-type lectins (CLECs) interact with $N$ - and $O$-linked glycans on GP, while PtdSer receptors interact with PtdSer present in the viral envelope (recently reviewed in $[31,32]$ ) (Fig. 1). These cell surface proteins do not serve as canonical enveloped virus receptors that interact with filovirus GP RBD amino acids. Instead, these proteins interact with filoviruses through more unconventional mechanisms discussed below. Outside the host cell, the filovirus GP RBD remains masked and conformationally unavailable for receptor interactions. It is only upon proteolytic processing within the endosomal compartment that more conventional filovirus GP/receptor interactions are made possible.

To date, CLECs, including dendritic cell-specific ICAM-3grabbing non-integrin (DC-SIGN), liver/lymph node-specific ICAM-3 grabbing non-integrin (L-SIGN), lymph node sinusoidal endothelial cell C-type lectin (LSECTin), asialoglycoprotein receptor 1 (ASGPRI), and human macrophage galactose- and acetylgalactosamine-specific C-type lectin (hMGL), have been identified to bind to $N$ - and $O$-linked glycans on EBOV GP to facilitate virus entry into a variety of cells [23•, 33-37]. DC-SIGN and L-SIGN are expressed on cells of the myeloid lineage and liver/lymph node endothelial cells, respectively. Several groups have demonstrated that high-mannose $N$-linked glycans on EBOV GP interact with DC/L-SIGN to facilitate entry [23•, 33, 38-41]. $N$ acetylglucosamine is important for EBOV entry mediated by LSECTin, which is expressed in sinusoidal endothelial cells in the liver and lymph nodes along with in vitro matured DCs and macrophages [35]. Mouse LSECTin, but not mouse DCSIGN, has been shown to mimic the properties of its human homolog, suggesting that mice lacking the LSECTin receptor could be utilized in infection studies to better define the role of 


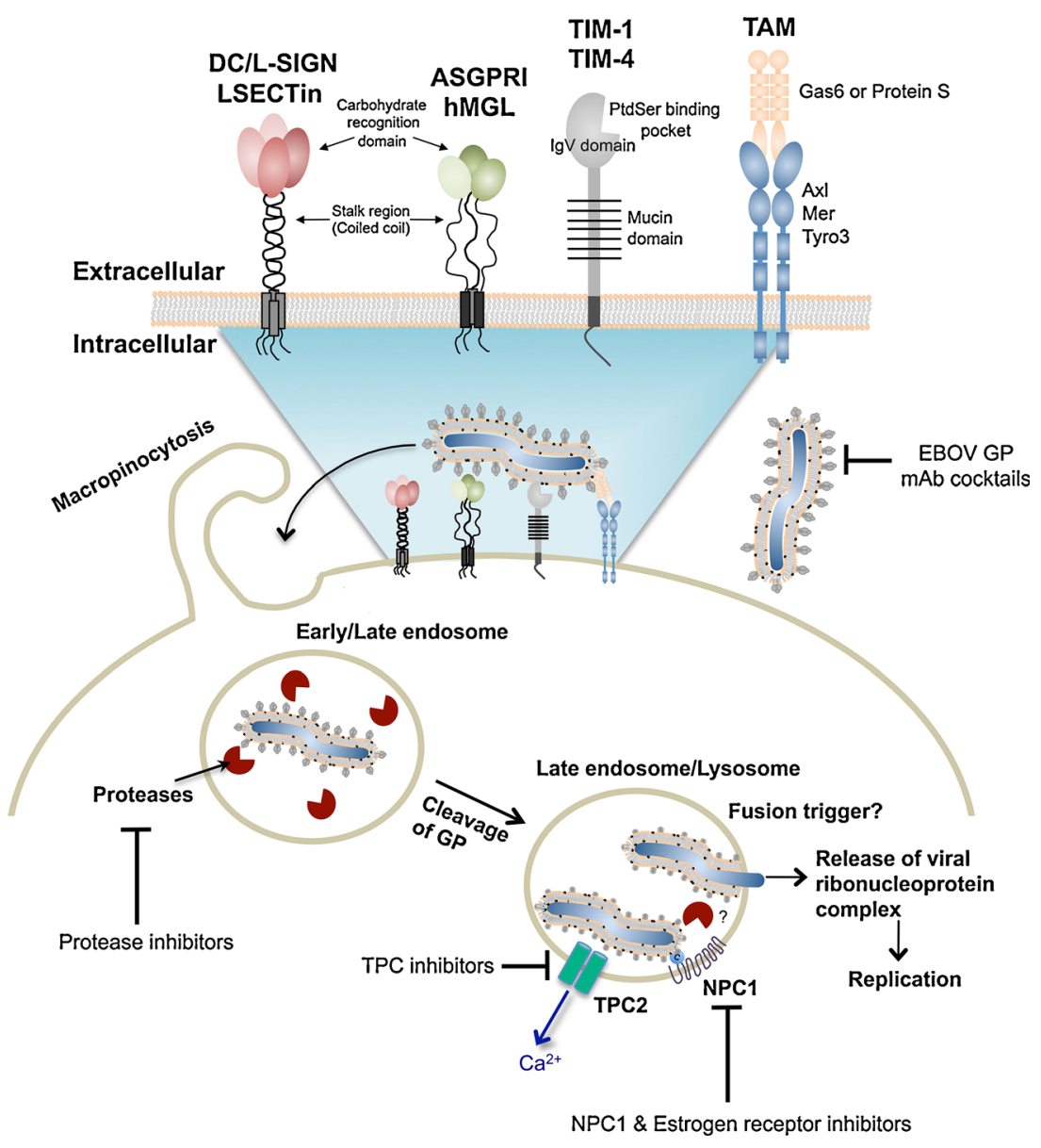

Fig. 1 Model of filovirus entry pathway. Filoviruses attach to the cell membrane via non-canonical cell surface receptors, C-type lectins (CLECs), and phosphatidylserine (PtdSer) receptors. CLECs (DC/LSIGN, LSECtin, ASGPRI, and hMGL) interact with sugars on the virion glycoprotein through their carbohydrate recognition domains. LSECtin is thought to exist as a dimer rather than a tetramer at the cell surface. PtdSer receptors (TIM and TAM family members) interact with PtdSer that is present in the virion envelope through their PtdSer binding pockets (TIM family) or through complexing with PtdSer binding proteins Gas6 or Protein S (TAM family). Following interaction with these cell surface proteins, the virions enter the endosomal pathway through a macropinocytosis-type uptake mechanism. Within the

this CLEC in vivo $[42,43]$. The other two CLEC family members known to enhance filovirus infection, ASGPRI and hMGL, are expressed on hepatocytes and monocyte-derived immature dendritic cells or macrophages, respectively. Both function as EBOV entry factors by binding specifically to galactose and $N$-acetylgalactosamine $[23 \cdot, 34,36]$.

The role of EBOV GP $N$-linked glycans on CLECdependent entry was recently evaluated [23•]. Elimination of $N$-linked glycans by mutagenesis of the MLD or the core and glycan cap of GP profoundly decreases virus entry mediated by cells ectopically expressing either DC-SIGN or L-SIGN. Not surprisingly, given these observations, the combined removal of all $N$-linked glycans on GP1 abrogated transduction by these CLECs, demonstrating the importance of these endosomal compartment, filovirus GP is cleaved by cysteine proteases, including cathepsin $\mathrm{B}$ and $\mathrm{L}$, to expose the receptor-binding domain (RBD) of filovirus GP. Exposure of the RBD allows GP to interact with the luminal C-domain of the intracellular receptor, NPC1. Once the virion interacts with NPC1, two-pore $\mathrm{Ca}^{2+}$ channels (TPCs) play an important role in late entry events. Potential additional proteolysis events may also be required for fusion to occur, but the exact steps leading to fusion remain unknown. Fusion then releases the viral ribonucleoprotein complex into the cytoplasm for replication. The therapeutics that are currently under investigation for their ability to block filovirus entry are indicated at the steps that they are thought to inhibit

glycans for interactions with these CLECs. Interestingly, LSECtin-dependent entry was less affected by loss of $N$ linked glycans on the core and glycan cap, but loss of $N$-linked glycans on the MLD abolished LSECtin-dependent virus entry. The impact of EBOV GP $N$-linked glycans on ASGPRIor hMGL-dependent entry has a quite different profile. Removal of $N$-glycans from the MLD, but not the core of GP, decreased EBOV entry mediated by ASGPR 1 about two-fold, and entry by hMGL was only modestly affected by GP1 $\mathrm{N}$-linked glycan loss. These findings providing evidence that MLD $O$-linked glycans, rather than the EBOV GP1 $N$-linked glycans, are critical for interaction with these CLECs.

A subset of PtdSer receptors have been shown to enhance entry of a variety of enveloped viruses, such as filoviruses, 
alphaviruses, and flaviviruses by binding to virionassociated PtdSer. To date, six PtdSer-mediated virus-entryenhancing receptors (PVEERs) have been identified. T-cell immunoglobulin and mucin domain (TIM)-1 and -4 directly bind to PtdSer on virions, whereas the Tyro3 (TAM) family tyrosine kinase receptors (Tyro3, Axl, and Mer) complex with Gas6 or Protein S to bind to PtdSer [7•, 8•, 44-46]. A complex composed of integrin $\alpha \mathrm{V} / \beta 3$ or $\beta 5$ is another PVEER that has been shown to facilitate uptake of lentiviral particles bearing a modified Sindbis virus GP [46]. Details of PtdSer receptors in virus entry were recently reviewed by Moller-Tank and Maury [32].

Although CLECs and PVEERs have been demonstrated to enhance virus attachment and infection, the specific mechanism(s) by which these factors induce internalization of virus particles remains unknown. Also, the relative importance of each CLEC or PVEER in vivo has yet to be explored. It remains unknown as to whether the loss of one of these proteins impacts filovirus infection or if these proteins are sufficiently redundant in their function that loss of them individually would have little consequence to virus entry. Determining the importance of these receptors in vivo may allow for the development of antibodies or small molecules to inhibit filovirus infection at cellular attachment.

\section{Filovirus Internalization and Endosomal Trafficking}

Following attachment, virions are internalized into endosomal compartments. Macropinocytosis is thought to be the primary uptake mechanism, but other routes of uptake, including caveolin- and clathrin-dependent endocytosis, have been also been reported [45, 47-53] (recently reviewed in [54]). It remains possible that the uptake mechanism used may be cell type dependent and/or cell surface receptor dependent. Differences in cell-dependent mechanisms of entry are supported by the observation that African green monkey epithelial cells (Vero) and human neuroblastoma cells (SNB19) primarily use macropinocytosis for viral uptake, but the signaling pathways required for uptake differ [45, 47, 49]. Filovirus entry in Vero cells is primarily, if not exclusively, mediated by TIM-1 and requires signaling through the phosphoinositide 3-kinase (PI3K)/Akt pathway [8•, 45, 55, 56]. In contrast, for entry into SNB-19 cells, surface expression of the TAM tyrosine kinase, Axl, and phospholipase C signaling is required [45]. It is not currently known if the requisite signaling is directly tied to virion interactions with specific receptors or if the signaling requirements are downstream from initial cell surface interactions. Recently, we have also observed that AMP-activated protein kinase (AMPK) is important for one or more early steps of EBOV entry in a variety of different cell types, but the detailed role of AMPK in entry remains to be elucidated [57].
Evidence suggests that filoviruses traffic from the cell surface, through early endosomes and into the late endosomal or lysosomal compartment before viral membranes fuse with cellular membranes to mediate viral genome entry into the cytoplasm $[58,59]$. A recent study demonstrated that release of the viral genome is slow, presumably due to the need for EBOV to traffic to late endosomal/lysosomal compartments [60•]. Upon initial internalization, EBOV colocalizes with early endosomal antigen-1 (EEA1) positive vesicles [47]. Rab5 and Rab7 GTPases are involved in trafficking the virions to late endosomes. At later times of entry, virions localize with perinuclear Rab7/LAMP-1-positive late endosomes, and a dominantnegative inhibitor of Rab7 reduces infection, suggesting the importance of Rab7 in EBOV entry [47]. Additional GTPases have also been implicated in EBOV endocytosis, including RhoB, Rac1, and CDC42 [49, 61]. Along with the involvement of GTPases in endosomal trafficking, other host protein and signaling pathways have been suggested to be involved in virion trafficking, including homotypic fusion and vacuole protein-sorting (HOPS) multi-subunit tethering complex and calciumcalmodulin kinases [55, 62, 63]. Required events between filovirus attachment and fusion are numerous and remain incompletely characterized; we have yet to fully map the endosomal route(s) that filoviruses travel to reach the site of viral fusion.

\section{Cathepsin Cleavage of Filovirus GP}

Filovirus GPs require proteolytic cleavage to expose the RBD, generating a fusion-ready form of the protein [19, 64, 65]. For EBOV, this event occurs within the endosomal/lysosomal pathway as early endosomes mature to late endosomes, acidifying the compartment and activating endosomal cysteine proteases, cathepsin $\mathrm{L}$ and $\mathrm{B}$. These low-pH-dependent proteases remove the heavily glycoslated MLD and glycan cap from GP1 to produce a 17- to 19-kDa protein [66-69]. Interestingly, other proteases, such as bacterial thermolysin, can effectively substitute for these cathepsins both in vitro and in vivo $[67,70 \bullet, 71 \bullet \bullet$. Early studies indicated that cathepsin L was not absolutely required for filovirus infection [64, 72]. Subsequent studies by Misasi et al. demonstrated that the infectivity of Marburgvirus as well as some Ebolavirus species also do not require cathepsin $\mathrm{B}$ in vitro, although proteolytic processing by one or more cysteine proteases is needed [70•]. Most recently, Marzi et al. demonstrated that both survival and viral organ titers from cathepsin B and L knockout mice following lethal challenge with mouse-adapted EBOV were similar to wild-type mice, indicating that loss of either of these cathepsins does not abrogate filovirus infection in vivo [71••]. In total, these studies indicate that EBOV GP proteolytic processing is a required step in the entry process, and, while cathepsins $\mathrm{B}$ and $\mathrm{L}$ can mediate efficient processing of some filoviruses, these proteases are neither specifically nor absolutely required. 
Once GP1 is proteolycally cleaved to expose the RBD, EBOV GP is able to interact with its intracellular receptor, NPC1 $[63,73,74 \bullet]$. Interestingly, several studies suggest that entry of proteolytically processed EBOV remains sensitive to broad-spectrum cysteine protease inhibitors, such as E64d, suggesting that in addition to the formation of the 17$19 \mathrm{kDa}$ form of the GP, there are additional proteaserequiring steps needed for fusion to occur $[60 \bullet, 64]$.

\section{Intracellular Receptor, NPC1, and the Role of Endosomal $\mathrm{Ca}^{2+}$ Channels}

In 2011, NPC1 was identified as a host protein required for filovirus entry by two independent screening studies $[63,73]$ (recently reviewed in $[58,75,76]$ ). NPC1 is a large multi-pass membrane protein that is a marker of specific late endosomal/ lysosomal compartments [77]. The physiological role of this protein is in trafficking of cholesterol $[78,79]$. The role of NPC1 in EBOV entry is independent of its role in cholesterol trafficking since cells lacking NPC2 or an NPC1 mutant defective in cholesterol trafficking are still able to mediate EBOV infection. Consistent with this, NPC1-deficient cells or biochemical knockdown of NPC1 results in inhibition of EBOV entry in tissue culture [63, 73]. Furthermore, NPC1 heterozygote mice displayed decreased mortality compared to wild-type mice when challenged with mouse-adapted EBOV [63]. NPC1 interacts only with the proteolytically cleaved GP specifically through the luminal $\mathrm{C}$ domain of NPC1 $[73,74 \cdot, 80]$. Most recently, it has been shown that EBOV VLP trafficking to the NPC1-positive late endosome/ lysosomes temporally correlates with viral/cellular membrane fusion events, suggesting that entry into this compartment is a key rate-defining step for EBOV entry and that fusion may occur with this vesicle [60•]. Additionally, a recent study suggests that upon TIM-1-dependent entry of EBOV particles, NPC1 colocalizes with TIM-1 in the same endosomal compartment, consistent with a possible virion "hand-off" between these two receptors as has been proposed [75, 81].

As the importance of NPC1 in filovirus entry was defined, parallel work established a role for the L-type calcium channels in entry $[82,83 \bullet \cdot$. Specifically, L-type channel drugs verapamil, tetrandrine, nimodipine, and diltiazem that inhibit calcium signaling induced by nicotinic acid adenine dinucleotide phosphate (NAADP) block EBOV infection [84]. These channels, known as two-pore $\mathrm{Ca}^{2+}$ channels (TPCs), are found in both $\mathrm{NPC} 1+$ and NPC1 - late endosomal compartments that are also LAMP1+. In addition, active TPCs are required for EBOV fusion [83••]. EBOV particles accumulate in TPC2+/NPC1+ compartments upon tetrandrine treatment, leading the authors to suggest that the TPC2+/NPC1- compartment is downstream from the double-positive compartment and may serve as the vesicle from which virions are released into the cytoplasm.

\section{Virus Fusion}

While the trigger of membrane fusion remains unknown, fusion of EBOV virions in the endosomal/lysosomal compartment is thought to be similar to those of other viruses with class I fusion GPs [29]. Conformational changes and proteolytic processing of GP expose the fusion loop in GP2, resulting in insertion of fusion loop hydrophobic residues into the vesicular membrane $[65,85]$. Recent studies have demonstrated that the conformation of the hydrophobic tip of the loop changes under low $\mathrm{pH}$ conditions, which presumably exist in this vesicular environment $[30,86 \bullet]$. Mutations of two hydrophobic residues in the fusion loop (I544 and L529) compromise virus fusion, suggesting that these residues are critical for forming a consolidated hydrophobic surface at the tip of the loop [86•]. Upon loop insertion into the vesicular membrane, the unwinding of the GP2 trimer causes refolding of the helical regions into a sixhelix bundle pulling the host and viral membranes into proximity for fusion [87]. Fusion allows for release of the viral RNA and associated viral proteins into the cytoplasm for downstream viral processes (Fig. 1).

\section{Therapeutics Targeting Filovirus Entry}

Small-Molecule Inhibitors In the search for effective therapeutics that broadly or specifically block filovirus family members, many groups have explored the ability of small molecule inhibitors to interfere with specific steps of the viral entry process (recently reviewed in [88]). The drugs being explored currently target events that occur within endosomes, such as the proteolysis of filovirus GP, endosomal trafficking, interactions with NPC1, and fusion. In addition, several nonspecific cysteine protease (E-64, leupeptin) or cathepsin B/L inhibitors (CA-074, FY-DMK, and CID23631927) have been investigated for their ability to inhibit EBOV infection in vitro [66, 67, 89-91]. Recently, another cysteine protease inhibitor, K11777, was identified to inhibit EBOV entry in tissue culture in sub-nanomolar concentrations and to be effective and safe in a SARS-CoV mouse infection [92]. The effectiveness of these protease inhibitors in vivo remains to be elucidated. However, the efficacy of some of these compounds may not translate to in vivo studies since as noted above cathepsins B and $\mathrm{L}$ are dispensable for in vivo EBOV replication. Consequently, inhibitors specific for these enzymes are not likely to prove efficacious against filoviruses in vivo [71••].

Several drugs have been investigated for their ability to inhibit EBOV entry through targeting late endosomal events, including NPC1 interactions. U18666A, a cationic amphiphile, induces a loss of NPC1 function by halting cholesterol transport, and through a poorly understood mechanism reduces EBOV infectivity in vitro $[93,94]$. Other cationic amphiphiles were shown to have a similar effect on both EBOV 
entry and cholesterol accumulation, providing evidence that a broad spectrum of these compounds have at least some efficacy against EBOV entry [95]. For example, the tertiary amine imipramine that interferes with both cholesterol/ sphingomyelin transport and inhibits acid sphingomyelinase activity reduces EBOV infectivity in vitro $[63,95,96]$. A third compound, 3.47, has also been shown to be effective in inhibiting EBOV entry in vitro and has been reported to directly interact with NPC1 $[73,97]$.

A set of related estrogen receptor inhibitors that are also cationic amphiphiles have been identified through an in vitro screen of FDA-approved molecules for their efficacy against EBOV [98]. Within this panel of drugs, clomiphene and toremifene were shown to protect against mouse-adapted EBOV in vivo. These selective estrogen receptor modulators inhibit EBOV by a mechanism independent of their ability to interact with estrogen and seemed to affect late entry events, perhaps in a manner similar to U18666A. The ability of these drugs to become potential human filovirus therapeutics relies on future work focusing on determining a detailed mechanism of viral inhibition along with determining their efficacy in vivo.

Calcium-calmodulin kinases were initially identified in a siRNA screen as host proteins critical for EBOV infection [62]. Calmodulin (CAMK2) inhibitor KN-93 profoundly inhibited entry of EBOV GP pseudotyped lentiviral vectors and reduced wild-type EBOV viral titers by $>95 \%$. Verapamil, tetradrine, nimodipine, and diltiazem inhibit TPCs found in the late endosome/lysosome compartment as discussed above. Mice treated with tetradrine have a significant decrease in morbidity and mortality and have reduced viral titers [83••]. These TPC inhibitors need further study but show promising antiviral efficacy.

\section{Monoclonal Antibody Therapies Against EBOV Entry Re-} cently, antibodies that bind to EBOV GP have been shown to be effective in protecting NHPs against lethal EBOV challenge $[99,100,101 \bullet \cdot$. A neutralizing monoclonal antibody (mAb), KZ52, was isolated from a human EBOV survivor and was utilized in crystallography studies that elucidated the EBOV GP structure [19, 102]. KZ52 binds the base of the pre-fusion GP1/GP2 and neutralizes infection in vitro [19, 20]. This region of GP has been termed a "hot spot" for neutralization due to the identification of a number of monoclonal antibodies that bind to this region including an anti-SUDV antibody 16F6 [20, 85]. Interestingly, KZ52 alone protects mice and guinea pigs against lethal infection but does not protect NHPs [103, 104].

More recently, it is combinations of different anti-EBOV GP mAbs that have been shown to protect NHPs against lethal challenge with EBOV when administered following infection [99, $100,101 \cdot \bullet]$. One effective cocktail is MB-003 (MappBio) that includes the anti-GP antibodies 13C6, 13F6, and 6D8 [99]. The other is ZMAb (Defyrus) that includes anti-GP antibodies $1 \mathrm{H} 3$, 2G4, and 4G7 [100,105•]. These two cocktails have been combined to make ZMapp (13C6, 2G4, and 4G7) that is being developed as an EBOV therapeutic [101••]. In mapping the epitopes of these antibodies, it has been shown that $13 \mathrm{C} 6$ and $1 \mathrm{H} 3$ bind to the glycan cap of GP1, whereas $2 \mathrm{G} 4$ and $4 \mathrm{G} 7$ bind at the GP1/GP2 interface in a similar region as KZ52 and 16 F6 and the latter $\mathrm{mAbs}$ within the cocktail effectively neutralize virus infection in vitro $[105 \bullet, 106 \bullet$. These studies demonstrate that a combination of antibodies that target the GP1-GP2 interface and the glycan cap of EBOV GP effectively provides protection in vivo.

Many mechanistic questions about how these antibodies are working to inhibit filovirus infection remain unanswered. Future work should explore the ability of these cocktails to protect against other Ebolavirus species and to develop similar cocktails against the other species if the current cocktails prove ineffective. Furthermore, studies are needed to determine the mechanism of protection provided by the glycan cap antibodies, and investigate the ability of these antibodies to interact with soluble GP. Additionally, and perhaps most importantly, we need to determine how readily the virus can evolve mutations within targeted epitopes, thereby escaping from inhibition by these antibodies.

\section{Conclusion}

Recent advances in filovirus entry research have provided valuable insights for the development of new therapeutics. In the past 5 years, the field has elucidated several steps within the complicated filovirus entry pathway. These include (1) identification of PtdSer receptors that serve as non-specific viral envelope attachment/internalization factors; (2) identification of NPC1 as a novel and essential endosomal receptor for filoviruses; (3) establishing the role of L-type calcium channels in entry; and (4) more in-depth knowledge of filovirus GP expression, structure, and function to better therapeutically target the entry pathway.

Our understanding of the details of the filovirus entry process is not yet complete. Future work will explore the mechanistic details of these entry steps and the proteins involved in vitro along with defining their critical roles during in vivo infection. Some important questions that still remain to be answered include as follows: What triggers viral internalization following viral attachment to the cell? Does the virus always follow the same endosomal compartment pathway? What host signaling pathways are involved in viral internalization and trafficking and can these pathways be targeted by therapeutics? Since not all filoviruses require cathepsin B and L, what other proteases can process GP? Also, what exact role does NPC1 play in fusion? What is/are the fusion trigger(s) that have yet to be identified? Through further development of a clear understanding of the basic cellular and virus biology underlying the entry process, we will be able to develop more effective and safe therapeutics to halt human filovirus infections. 


\section{Compliance with Ethics Guidelines}

Conflict of Interest Statement The authors declare that they have no conflicts of interests

Human and Animal Rights and Informed Consent This article contains no studies with human or animal subjects performed by the author.

\section{References}

Papers of particular interest, published recently, have been highlighted as:

- Of importance

•- Of major importance

1. Kuhn JH, Becker S, Ebihara H, Geisbert TW, Johnson KM, Kawaoka Y, et al. Proposal for a revised taxonomy of the family Filoviridae: classification, names of taxa and viruses, and virus abbreviations. Arch Virol. 2010;155(12):2083-103.

2. Feldmann H, Geisbert TW. Ebola haemorrhagic fever. Lancet. 2011;377(9768):849-62.

3. Centers for Disease Control and Prevention 2014 Ebola outbreak in West Africa[May 11, 2015]. Available from http://www.cdc. gov/vhf/ebola/outbreaks/2014-west-africa/index.html.

4. Leroy EM, Gonzalez JP, Baize S. Ebola and Marburg haemorrhagic fever viruses: major scientific advances, but a relatively minor public health threat for Africa. Clin Microbiol Infect. 2011;17(7):964-76.

5. Martines RB, Ng DL, Greer PW, Rollin PE, Zaki SR. Tissue and cellular tropism, pathology and pathogenesis of Ebola and Marburg viruses. J Pathol. 2015;235(2):153-74.

6. Geisbert TW, Hensley LE, Gibb TR, Steele KE, Jaax NK, Jahrling PB. Apoptosis induced in vitro and in vivo during infection by Ebola and Marburg viruses. Lab Investig; J Techn Method Pathol. 2000;80(2):171-86.

7. Jemielity S, Wang JJ, Chan YK, Ahmed AA, Li W, Monahan S, et al. TIM-family proteins promote infection of multiple enveloped viruses through virion-associated phosphatidylserine. PLoS Pathog. 2013;9(3):e1003232. Identified that TIM and TAM proteins bind to PtdSer in virion envelopes to promote infection of a wide array of enveloped viruses including filoviruses, flaviviruses, New World arenaviruses and alphaviruses.

8. Moller-Tank S, Kondratowicz AS, Davey RA, Rennert PD, Maury W. Role of the phosphatidylserine receptor TIM-1 in envelopedvirus entry. J Virol. 2013;87(15):8327-41. Defined the molecular interactions between TIM-1 and PtdSer on the surface of Ebola virus virions. They also demonstrated that TIM-1 can enhance infection of alphavirus and baculovirus families.

9. Noda T, Kolesnikova L, Becker S, Kawaoka Y. The importance of the NP: VP35 ratio in Ebola virus nucleocapsid formation. J Infect Dis. 2011;204 Suppl 3:S878-83.

10. Becker S, Rinne C, Hofsass U, Klenk HD, Muhlberger E. Interactions of Marburg virus nucleocapsid proteins. Virology. 1998;249(2):406-17.

11. Groseth A, Charton JE, Sauerborn M, Feldmann F, Jones SM, Hoenen T, et al. The Ebola virus ribonucleoprotein complex: a novel VP30-L interaction identified. Virus Res. 2009;140(1-2):8-14.

12. Hoenen T, Jung S, Herwig A, Groseth A, Becker S. Both matrix proteins of Ebola virus contribute to the regulation of viral genome replication and transcription. Virology. 2010;403(1):56-66.
13. Harrison SC. Viral membrane fusion. Virology. 2015. doi:: 10.1016/j.virol.2015.03.043

14. Sanchez A, Yang ZY, Xu L, Nabel GJ, Crews T, Peters CJ. Biochemical analysis of the secreted and virion glycoproteins of Ebola virus. J Virol. 1998;72(8):6442-7.

15. Audet J, Kobinger GP. Immune evasion in ebolavirus infections. Viral Immunol. 2015;28(1):10-8.

16. Sanchez A, Trappier SG, Mahy BW, Peters CJ, Nichol ST. The virion glycoproteins of Ebola viruses are encoded in two reading frames and are expressed through transcriptional editing. Proc Natl Acad Sci U S A. 1996;93(8):3602-7.

17. Feldmann H, Volchkov VE, Volchkova VA, Klenk HD. The glycoproteins of Marburg and Ebola virus and their potential roles in pathogenesis. Arch Virol Suppl. 1999;15:159-69.

18. Volchkov VE, Feldmann H, Volchkova VA, Klenk HD. Processing of the Ebola virus glycoprotein by the proprotein convertase furin. Proc Natl Acad Sci U S A. 1998;95(10):5762-7.

19. Lee JE, Fusco ML, Hessell AJ, Oswald WB, Burton DR, Saphire EO. Structure of the Ebola virus glycoprotein bound to an antibody from a human survivor. Nature. 2008;454(7201):177-82.

20. Dias JM, Kuehne AI, Abelson DM, Bale S, Wong AC, Halfmann $\mathrm{P}$, et al. A shared structural solution for neutralizing ebolaviruses. Nat Struct Mol Biol. 2011;18(12):1424-7.

21.• Hashiguchi T, Fusco ML, Bornholdt ZA, Lee JE, Flyak AI, Matsuoka R, et al. Structural basis for Marburg virus neutralization by a cross-reactive human antibody. Cell. 2015;160(5):904 12. Determined the crystal structure of Marburg virus GP utilizing antibodies from a human survivor. They identified that the mucin-containing domain of MARV is arranged differently that that domain in EBOV. This explains why antibodies targeting the base of MARV GP1 have yet to be identified.

22. Feldmann H, Nichol ST, Klenk HD, Peters CJ, Sanchez A. Characterization of filoviruses based on differences in structure and antigenicity of the virion glycoprotein. Virology. 1994;199(2):469-73.

23. Lennemann NJ, Rhein BA, Ndungo E, Chandran K, Qiu X, Maury W. Comprehensive functional analysis of N-linked glycans on Ebola virus GP1. mBio. 2014;5(1):e00862-13. Demonstrated the importance of $\mathrm{N}$-linked and $\mathrm{O}$-linked glycans of the EBOV GP MLD, core and glycan cap in C-type lectin mediated $\mathrm{EBOV}$ entry as well as the other role these glycans play in the EBOV biology.

24. Wilson JA, Hevey M, Bakken R, Guest S, Bray M, Schmaljohn AL. Epitopes involved in antibody-mediated protection from Ebola virus. Science (New York, NY). 2000;287(5458):1664-6.

25. Dowling W, Thompson E, Badger C, Mellquist JL, Garrison AR, Smith JM, et al. Influences of glycosylation on antigenicity, immunogenicity, and protective efficacy of ebola virus GP DNA vaccines. J Virol. 2007;81(4):1821-37.

26. Martinez O, Tantral L, Mulherkar N, Chandran K, Basler CF. Impact of Ebola mucin-like domain on antiglycoprotein antibody responses induced by Ebola virus-like particles. J Infect Dis. 2011;204 Suppl 3:S825-32.

27. Ito H, Watanabe S, Sanchez A, Whitt MA, Kawaoka Y. Mutational analysis of the putative fusion domain of Ebola virus glycoprotein. J Virol. 1999;73(10):8907-12.

28. Jeffers SA, Sanders DA, Sanchez A. Covalent modifications of the ebola virus glycoprotein. J Virol. 2002;76(24):12463-72.

29. White JM, Delos SE, Brecher M, Schornberg K. Structures and mechanisms of viral membrane fusion proteins: multiple variations on a common theme. Crit Rev Biochem Mol Biol. 2008;43(3):189-219.

30. Gregory SM, Harada E, Liang B, Delos SE, White JM, Tamm LK. Structure and function of the complete internal fusion loop from 
Ebolavirus glycoprotein 2. Proc Natl Acad Sci U S A. 2011;108(27):11211-6.

31. Mason CP, Tarr AW. Human lectins and their roles in viral infections. Molecules (Basel, Switzerland). 2015;20(2):2229-71.

32. Moller-Tank S, Maury W. Phosphatidylserine receptors: enhancers of enveloped virus entry and infection. Virology. 2014;468-470:565-80.

33. Alvarez CP, Lasala F, Carrillo J, Muniz O, Corbi AL, Delgado R. C-type lectins DC-SIGN and L-SIGN mediate cellular entry by Ebola virus in cis and in trans. J Virol. 2002;76(13):6841-4.

34. Takada A, Fujioka K, Tsuiji M, Morikawa A, Higashi N, Ebihara $\mathrm{H}$, et al. Human macrophage C-type lectin specific for galactose and $\mathrm{N}$-acetylgalactosamine promotes filovirus entry. J Virol. 2004;78(6):2943-7.

35. Powlesland AS, Fisch T, Taylor ME, Smith DF, Tissot B, Dell A, et al. A novel mechanism for LSECtin binding to Ebola virus surface glycoprotein through truncated glycans. J Biol Chem. 2008;283(1):593-602.

36. Becker S, Spiess M, Klenk HD. The asialoglycoprotein receptor is a potential liver-specific receptor for Marburg virus. J Gen Virol. 1995;76(Pt 2):393-9.

37. Dahlmann F, Biedenkopf N, Babler A, Jahnen-Dechent W, Karsten CB, Gnirss K, et al. Analysis of Ebola virus entry into macrophages. J Infect Dis. 2015. doi: 10.1093/infdis/jiv140

38. Marzi A, Akhavan A, Simmons G, Gramberg T, Hofmann H, Bates $\mathrm{P}$, et al. The signal peptide of the ebolavirus glycoprotein influences interaction with the cellular lectins DC-SIGN and DCSIGNR. J Virol. 2006;80(13):6305-17.

39. Marzi A, Moller P, Hanna SL, Harrer T, Eisemann J, Steinkasserer A, et al. Analysis of the interaction of Ebola virus glycoprotein with DC-SIGN (dendritic cell-specific intercellular adhesion molecule 3-grabbing nonintegrin) and its homologue DC-SIGNR. J Infect Dis. 2007;196 Suppl 2:S237-46.

40. Simmons G, Reeves JD, Grogan CC, Vandenberghe LH, Baribaud F, Whitbeck JC, et al. DC-SIGN and DC-SIGNR bind ebola glycoproteins and enhance infection of macrophages and endothelial cells. Virology. 2003;305(1):115-23.

41. Lin G, Simmons G, Pohlmann S, Baribaud F, Ni H, Leslie GJ, et al. Differential N-linked glycosylation of human immunodeficiency virus and Ebola virus envelope glycoproteins modulates interactions with DC-SIGN and DCSIGNR. J Virol. 2003;77(2):1337-46.

42. Gramberg T, Caminschi I, Wegele A, Hofmann H, Pohlmann S. Evidence that multiple defects in murine DC-SIGN inhibit a functional interaction with pathogens. Virology. 2006;345(2):482-91.

43. Pipirou Z, Powlesland AS, Steffen I, Pohlmann S, Taylor ME, Drickamer K. Mouse LSECtin as a model for a human Ebola virus receptor. Glycobiology. 2011;21(6):806-12.

44. Shimojima M, Takada A, Ebihara H, Neumann G, Fujioka K, Irimura $\mathrm{T}$, et al. Tyro3 family-mediated cell entry of Ebola and Marburg viruses. J Virol. 2006;80(20):10109-16.

45. Hunt CL, Kolokoltsov AA, Davey RA, Maury W. The Tyro3 receptor kinase Axl enhances macropinocytosis of Zaire ebolavirus. J Virol. 2011;85(1):334-47.

46. Morizono K, Chen IS. Role of phosphatidylserine receptors in enveloped virus infection. J Virol. 2014;88(8):4275-90.

47. Saeed MF, Kolokoltsov AA, Albrecht T, Davey RA. Cellular entry of ebola virus involves uptake by a macropinocytosis-like mechanism and subsequent trafficking through early and late endosomes. PLoS Pathog. 2010;6(9):e1001110.

48. Empig CJ, Goldsmith MA. Association of the caveola vesicular system with cellular entry by filoviruses. J Virol. 2002;76(10): 5266-70.

49. Nanbo A, Imai M, Watanabe S, Noda T, Takahashi K, Neumann $\mathrm{G}$, et al. Ebolavirus is internalized into host cells via macropinocytosis in a viral glycoprotein-dependent manner. PLoS Pathog. 2010;6(9):e1001121.

50. Bhattacharyya S, Warfield KL, Ruthel G, Bavari S, Aman MJ, Hope TJ. Ebola virus uses clathrin-mediated endocytosis as an entry pathway. Virology. 2010;401(1):18-28.

51. Aleksandrowicz P, Marzi A, Biedenkopf N, Beimforde N, Becker $\mathrm{S}$, Hoenen $\mathrm{T}$, et al. Ebola virus enters host cells by macropinocytosis and clathrin-mediated endocytosis. J Infect Dis. 2011;204 Suppl 3:S957-67.

52. Mulherkar N, Raaben M, de la Torre JC, Whelan SP, Chandran K. The Ebola virus glycoprotein mediates entry via a non-classical dynamin-dependent macropinocytic pathway. Virology. 2011;419(2):72-83.

53. Bhattacharyya S, Hope TJ, Young JA. Differential requirements for clathrin endocytic pathway components in cellular entry by Ebola and Marburg glycoprotein pseudovirions. Virology. 2011;419(1):1-9.

54. Bhattacharyya S, Mulherkar N, Chandran K. Endocytic pathways involved in filovirus entry: advances, implications and future directions. Viruses. 2012;4(12):3647-64.

55. Saeed MF, Kolokoltsov AA, Freiberg AN, Holbrook MR, Davey RA. Phosphoinositide-3 kinase-Akt pathway controls cellular entry of Ebola virus. PLoS Pathog. 2008;4(8):e1000141.

56. Kondratowicz AS, Lennemann NJ, Sinn PL, Davey RA, Hunt CL, Moller-Tank S, et al. T-cell immunoglobulin and mucin domain 1 (TIM-1) is a receptor for Zaire Ebolavirus and Lake Victoria Marburgvirus. Proc Natl Acad Sci U S A. 2011;108(20):8426-31.

57. Kondratowicz AS, Hunt CL, Davey RA, Cherry S, Maury WJ. AMP-activated protein kinase is required for the macropinocytic internalization of ebolavirus. J Virol. 2013;87(2):746-55.

58. Miller EH, Chandran K. Filovirus entry into cells - new insights. Curr Opin Virol. 2012;2(2):206-14.

59. Moller-Tank S, Maury W. Ebola virus entry: a curious and complex series of events. PLoS Pathog. 2015;11(4):e1004731.

60. Mingo RM, Simmons JA, Shoemaker CJ, Nelson EA, Schornberg KL, D'Souza RS, et al. Ebola virus and severe acute respiratory syndrome coronavirus display late cell entry kinetics: evidence that transport to NPC1+ endolysosomes is a rate-defining step. J Virol. 2015;89(5):2931-43. Provided evidence of the slow kinetics of EBOV entry. Also demonstrated that EBOV traffics late into the endocytic pathway to NPC1+ late endo/lysosomal vesicles, where there are higher levels of cathepsin activity, suggesting the need for additional proteolysis following NPC1 interaction for fusion to occur.

61. Quinn K, Brindley MA, Weller ML, Kaludov N, Kondratowicz A, Hunt CL, et al. Rho GTPases modulate entry of Ebola virus and vesicular stomatitis virus pseudotyped vectors. J Virol. 2009;83(19):10176-86.

62. Kolokoltsov AA, Saeed MF, Freiberg AN, Holbrook MR, Davey RA. Identification of novel cellular targets for therapeutic intervention against Ebola virus infection by siRNA screening. Drug Dev Res. 2009;70(4):255-65.

63. Carette JE, Raaben M, Wong AC, Herbert AS, Obernosterer G, Mulherkar N, et al. Ebola virus entry requires the cholesterol transporter Niemann-Pick C1. Nature. 2011;477(7364):340-3.

64. Wong AC, Sandesara RG, Mulherkar N, Whelan SP, Chandran K. A forward genetic strategy reveals destabilizing mutations in the Ebolavirus glycoprotein that alter its protease dependence during cell entry. J Virol. 2010;84(1):163-75.

65. Brecher M, Schornberg KL, Delos SE, Fusco ML, Saphire EO, White JM. Cathepsin cleavage potentiates the Ebola virus glycoprotein to undergo a subsequent fusion-relevant conformational change. J Virol. 2012;86(1):364-72.

66. Chandran K, Sullivan NJ, Felbor U, Whelan SP, Cunningham JM. Endosomal proteolysis of the Ebola virus glycoprotein is 
necessary for infection. Science (New York, NY). 2005;308(5728):1643-5.

67. Schornberg K, Matsuyama S, Kabsch K, Delos S, Bouton A, White J. Role of endosomal cathepsins in entry mediated by the Ebola virus glycoprotein. J Virol. 2006;80(8):4174-8.

68. Dube D, Brecher MB, Delos SE, Rose SC, Park EW, Schornberg $\mathrm{KL}$, et al. The primed ebolavirus glycoprotein (19-kilodalton GP1, 2 ): sequence and residues critical for host cell binding. J Virol. 2009;83(7):2883-91.

69. Hood CL, Abraham J, Boyington JC, Leung K, Kwong PD, Nabel GJ. Biochemical and structural characterization of cathepsin Lprocessed Ebola virus glycoprotein: implications for viral entry and immunogenicity. J Virol. 2010;84(6):2972-82.

70. Misasi J, Chandran K, Yang JY, Considine B, Filone CM, Cote M, et al. Filoviruses require endosomal cysteine proteases for entry but exhibit distinct protease preferences. J Virol. 2012;86(6): 3284-92. Characterized the cathepsin B and $L$ dependency of different EBOV species. Identified that different filovirus species have altered utilization of cathepsin $L$ and $B$ alone or in combination.

71.• Marzi A, Reinheckel T, Feldmann H. Cathepsin B \& L are not required for ebola virus replication. PLoS Negl Trop Dis. 2012;6(12):e1923. Demonstrated no differences in survival or morbidity of cathepsin B and $L$ single-knockout mice compared to wild-type mice following lethal EBOV challenge. These results suggest that neither of these proteases are specifically and absolutely required for in vivo EBOV infection.

72. Martinez O, Johnson J, Manicassamy B, Rong L, Olinger GG, Hensley LE, et al. Zaire Ebola virus entry into human dendritic cells is insensitive to cathepsin L inhibition. Cell Microbiol. 2010;12(2):148-57.

73. Cote M, Misasi J, Ren T, Bruchez A, Lee K, Filone CM, et al. Small molecule inhibitors reveal Niemann-Pick $\mathrm{C} 1$ is essential for Ebola virus infection. Nature. 2011;477(7364):344-8.

74. Miller EH, Obernosterer G, Raaben M, Herbert AS, Deffieu MS, Krishnan A, et al. Ebola virus entry requires the host-programmed recognition of an intracellular receptor. EMBO J. 2012;31(8): 1947-60. Identified that cleaved filovirus GP interacts directly with the luminal C-loop of NPC1 and this interaction is important for entry steps in the late endosome/lysosome.

75. Hunt CL, Lennemann NJ, Maury W. Filovirus entry: a novelty in the viral fusion world. Viruses. 2012;4(2):258-75.

76. White JM, Schornberg KL. A new player in the puzzle of filovirus entry. Nat Rev Microbiol. 2012;10(5):317-22.

77. van der Kant R, Zondervan I, Janssen L, Neefjes J. Cholesterolbinding molecules MLN64 and ORP1L mark distinct late endosomes with transporters ABCA3 and NPC1. J Lipid Res. 2013;54(8):2153-65.

78. Carstea ED, Morris JA, Coleman KG, Loftus SK, Zhang D, Cummings C. Niemann-Pick $\mathrm{C} 1$ disease gene: homology to mediators of cholesterol homeostasis. Science (New York, NY). 1997;277(5323):228-31.

79. Higgins ME, Davies JP, Chen FW, Ioannou YA. Niemann-Pick C1 is a late endosome-resident protein that transiently associates with lysosomes and the trans-Golgi network. Mol Genet Metab. 1999;68(1):1-13.

80. Krishnan A, Miller EH, Herbert AS, Ng M, Ndungo E, Whelan SP, et al. Niemann-Pick C1 (NPC1)/NPC1-like1 chimeras define sequences critical for NPC1's function as a flovirus entry receptor. Viruses. 2012;4(11):2471-84.

81. Kuroda M, Fujikura D, Nanbo A, Marzi A, Noyori O, Kajihara M, et al. The interaction between TIM-1 and NPC1 Is important for the cellular entry of Ebola virus. J Virol. 2015. doi: 10.1128/JVI.03156-14

82. Gehring G, Rohrmann K, Atenchong N, Mittler E, Becker S, Dahlmann F, et al. The clinically approved drugs amiodarone, dronedarone and verapamil inhibit filovirus cell entry. J Antimicrob Chemother. 2014;69(8):2123-31.

83.• Sakurai Y, Kolokoltsov AA, Chen CC, Tidwell MW, Bauta WE, Klugbauer N. Ebola virus. Two-pore channels control Ebola virus host cell entry and are drug targets for disease treatment. Science (New York, NY). 2015;347(6225):995-8. The first demonstration of the importance of L-type calcium channels in Ebola virus entry. They also demonstrate the ability of calcium channel inhibitors to protect mice against lethal Ebola virus challenge.

84. Genazzani AA, Mezna M, Dickey DM, Michelangeli F, Walseth TF, Galione A. Pharmacological properties of the Ca2+-release mechanism sensitive to NAADP in the sea urchin egg. Br J Pharmacol. 1997;121(7):1489-95.

85. Bale S, Dias JM, Fusco ML, Hashiguchi T, Wong AC, Liu T, et al. Structural basis for differential neutralization of ebolaviruses. Viruses. 2012;4(4):447-70.

86. Gregory SM, Larsson P, Nelson EA, Kasson PM, White JM, Tamm LK. Ebolavirus entry requires a compact hydrophobic fist at the tip of the fusion loop. J Virol. 2014;88(12):6636-49. Demonstrated that the conformation of the hydrophobic tip of the GP2 fusion loop changes under low $\mathrm{pH}$ conditions and identifies two residues in the fusion loop (I544 and L529) that are critical for forming a consolidated hydrophobic surface at the tip of the loop.

87. Weissenhorn W, Carfi A, Lee KH, Skehel JJ, Wiley DC. Crystal structure of the Ebola virus membrane fusion subunit, GP2, from the envelope glycoprotein ectodomain. Mol Cell. 1998;2(5):605-16

88. Picazo E, Giordanetto F. Small molecule inhibitors of ebola virus infection. Drug Discov Today. 2015;20(2):277-86.

89. Barrientos LG, Rollin PE. Release of cellular proteases into the acidic extracellular milieu exacerbates Ebola virus-induced cell damage. Virology. 2007;358(1):1-9.

90. Gnirss K, Kuhl A, Karsten C, Glowacka I, Bertram S, Kaup F, et al. Cathepsins B and L activate Ebola but not Marburg virus glycoproteins for efficient entry into cell lines and macrophages independent of TMPRSS2 expression. Virology. 2012;424(1):3-10.

91. Shah PP, Wang T, Kaletsky RL, Myers MC, Purvis JE, Jing H, et al. A small-molecule oxocarbazate inhibitor of human cathepsin L blocks severe acute respiratory syndrome and ebola pseudotype virus infection into human embryonic kidney $293 \mathrm{~T}$ cells. Mol Pharmacol. 2010;78(2):319-24.

92. Zhou Y, Vedantham P, Lu K, Agudelo J, Carrion Jr R, Nunneley $\mathrm{JW}$, et al. Protease inhibitors targeting coronavirus and filovirus entry. Antivir Res. 2015;116:76-84.

93. Cenedella RJ. Cholesterol synthesis inhibitor U18666A and the role of sterol metabolism and trafficking in numerous pathophysiological processes. Lipids. 2009;44(6):477-87.

94. Rodriguez-Lafrasse C, Rousson R, Bonnet J, Pentchev PG, Louisot P, Vanier MT. Abnormal cholesterol metabolism in imipramine-treated fibroblast cultures. Similarities with Niemann-Pick type C disease. Biochim Biophys Acta. 1990;1043(2):123-8.

95. Shoemaker CJ, Schornberg KL, Delos SE, Scully C, Pajouhesh H, Olinger GG, et al. Multiple cationic amphiphiles induce a Niemann-Pick $\mathrm{C}$ phenotype and inhibit Ebola virus entry and infection. PLoS One. 2013;8(2):e56265.

96. Miller ME, Adhikary S, Kolokoltsov AA, Davey RA. Ebolavirus requires acid sphingomyelinase activity and plasma membrane sphingomyelin for infection. J Virol. 2012;86(14):7473-83.

97. Lee K, Ren T, Cote M, Gholamreza B, Misasi J, Bruchez A, et al. Inhibition of Ebola virus infection: identification of Niemann-Pick $\mathrm{C} 1$ as the target by optimization of a chemical probe. ACS Med Chem Lett. 2013;4(2):239-43. 
98. Johansen LM, Brannan JM, Delos SE, Shoemaker CJ, Stossel A, Lear C. FDA-approved selective estrogen receptor modulators inhibit Ebola virus infection. Sci Transl Med. 2013;5(190):190ra79.

99. Pettitt J, Zeitlin L, Kim do H, Working C, Johnson JC, Bohorov O. Therapeutic intervention of Ebola virus infection in rhesus macaques with the MB-003 monoclonal antibody cocktail. Sci Transl Med. 2013;5(199):199ra13.

100. Qiu X, Audet J, Wong G, Pillet S, Bello A, Cabral T, et al. Successful treatment of ebola virus-infected cynomolgus macaques with monoclonal antibodies. Sci Transl Med. 2012;4(138):138ra81.

101.• Qiu X, Wong G, Audet J, Bello A, Fernando L, Alimonti JB, et al. Reversion of advanced Ebola virus disease in nonhuman primates with ZMapp. Nature. 2014;514(7520):47-53. Demonstrated the ability of the ZMapp antibody cocktail to protect $100 \%$ of rhesus macaques when treatment is performed up to 5 days following lethal EBOV challenge.

102. Maruyama T, Rodriguez LL, Jahrling PB, Sanchez A, Khan AS, Nichol ST, et al. Ebola virus can be effectively neutralized by antibody produced in natural human infection. J Virol. 1999;73(7):6024-30.
103. Parren PW, Geisbert TW, Maruyama T, Jahrling PB, Burton DR. Pre- and postexposure prophylaxis of Ebola virus infection in an animal model by passive transfer of a neutralizing human antibody. J Virol. 2002;76(12):6408-12.

104. Oswald WB, Geisbert TW, Davis KJ, Geisbert JB, Sullivan NJ, Jahrling PB, et al. Neutralizing antibody fails to impact the course of Ebola virus infection in monkeys. PLoS Pathog. 2007;3(1):e9.

105. Audet J, Wong G, Wang H, Lu G, Gao GF, Kobinger G, et al. Molecular characterization of the monoclonal antibodies composing ZMAb: a protective cocktail against Ebola virus. Scientif Rep. 2014;4:6881. Described the epitope binding properties of ZMAb antibodies 1H3, 2G4 and 4G7.

106. Murin CD, Fusco ML, Bornholdt ZA, Qiu X, Olinger GG, Zeitlin L, et al. Structures of protective antibodies reveal sites of vulnerability on Ebola virus. Proc Natl Acad Sci U S A. 2014;111(48):17182-7. Described the binding of mAbs from the ZMapp, MB-003 and ZMAb cocktails, identifying the targeted sites for antibody binding of the EBOV glycoprotein. 